\title{
Generation of a precise Oct4-hrGFP knockin cynomolgus monkey model via CRISPR/Cas9-assisted homologous recombination
}

\author{
Cell Research (2018) 28:383-386. doi:10.1038/cr.2018.10; published online 12 January 2018
}

\section{Dear Editor,}

The close genetic and physiological similarity to humans make non-human primates (NHPs) excellent models for biomedical research [1]. Genetically modified monkey models are invaluable for recapitulating human diseases. Reprogrammable nuclease CRISPR/Cas9 shows tremendous potentials for gene targeting in monkeys. The nuclease causes double-stranded breaks, which results in gene knockout when the breaks are repaired by non-homologous end-joining (NHEJ), or gene knockin when repaired by homology-directed repair (HDR) in the presence of exogenous templates [2]. We have previously generated CRISPR/Cas9-mediated cynomolgus monkeys [3]. However, currently achieved genetic targeting in monkeys is gene disruption by frame shift, which is unpredictable and uncontrollable. Thus, precise genome editing of endogenous loci is urgently needed to more faithfully model human diseases. Our group has achieved efficient CRISPR/Cas9-mediated precise genome editing in rats [4], which prompted us to extend the work to monkeys.

Oct4 is a critical regulator of pluripotency and a germ cell marker [5]. We aimed to generate Oct4-GFP knockin allele through insertion of a less toxic humanized recombinant green fluorescent protein ( $h r G F P$ ) sequence (Agilent, Cat\# 240031) into the last codon of Oct4 by Cas9/sgRNA-mediated HDR (Supplementary information, Figure S1). To generate Oct4-hrGFP knockin cynomolgus monkey, a donor plasmid containing a 1057 bp left homologous arm (L-HA) and a 1081 bp right homologous arm (R-HA) flanking the internal ribozyme entry site (IRES)-hrGFP sequence, Cas9 (Addgene, Cat\# 44758) mRNA, and sgRNA targeting the 3' UTR region around the stop codon of Oct4 (Supplementary information, Table S1) were pooled and microinjected into 198 zygotes. Among them, 120 developed normally and were transferred into 40 surrogate females, 12 pregnancies were established, and 8 live births were born subse- quently (Supplementary information, Tables S2 and S3). Genotyping was performed (Supplementary information, Tables S3 and S5). Among 8 aborted fetuses, hrGFP insertion sequence (Figure 1A) and together with $5^{\prime}$ and 3' homologous arms (Figure 1B; Supplementary information, Data S2 and S3) were successfully amplified by PCR in all the tested tissues of fetus J, and the knockin cassette was confirmed by sequencing the targeted region (Supplementary information, Figure S2 and Data S4), indicating the $h r G F P$ integration and the possible precise Oct4-hrGFP knockin in fetus J. Genomic DNAs from muscle $(\mathrm{Mu})$ and small intestine (SI) of fetus J were digested by $S t u I$ and analyzed by Southern blot using hrGFP internal probe (Probe 1), 5' external probe (Probe 2 ), and 3' external probe (Probe 3), respectively (Supplementary information, Table S4). As expected, Oct4-hrGFP integration produced a $4.7 \mathrm{~kb}$ mutant band in addition to a $3.4 \mathrm{~kb}$ wild-type band (Figure $1 \mathrm{C}$ ). Taken together, these results demonstrated that $h r G F P$ has been precisely integrated into the Oct4 locus in different tissues of fetus $\mathrm{J}$.

Of the 8 live births, one of the last two born founders $(152008,152018)$ was found to undergo genome targeting (Figure 1D). The genotyping analysis showed that placenta and umbilical cord from Founder 152008 yielded specific band of $h r G F P$ (Supplementary information, Figure S3) and homologous arms were detected as clear $3^{\prime}$-arm and $5^{\prime}$-arm bands (Figure 1E), and precise insertion by $5^{\prime}$ - and $3^{\prime}$-homologous recombination was confirmed by sequencing the amplified genomic DNA (Figure $1 \mathrm{~F}$ and Supplementary information, Data S5 and S6). Besides the precise knockin in Founder 152008, the imprecise genome targeting events occurred in both founders (152008 and 152018) as shown by distinct T7EN1 cleavage bands (Supplementary information, Figure S4A and Table S5). Similar to fetus J, an extra bigger homologous recombination band from knockin sequence was detected in the knockin monkey 152008 (Supplementary information, Figure S4A and Data S7). Of note, as in fetus J, 


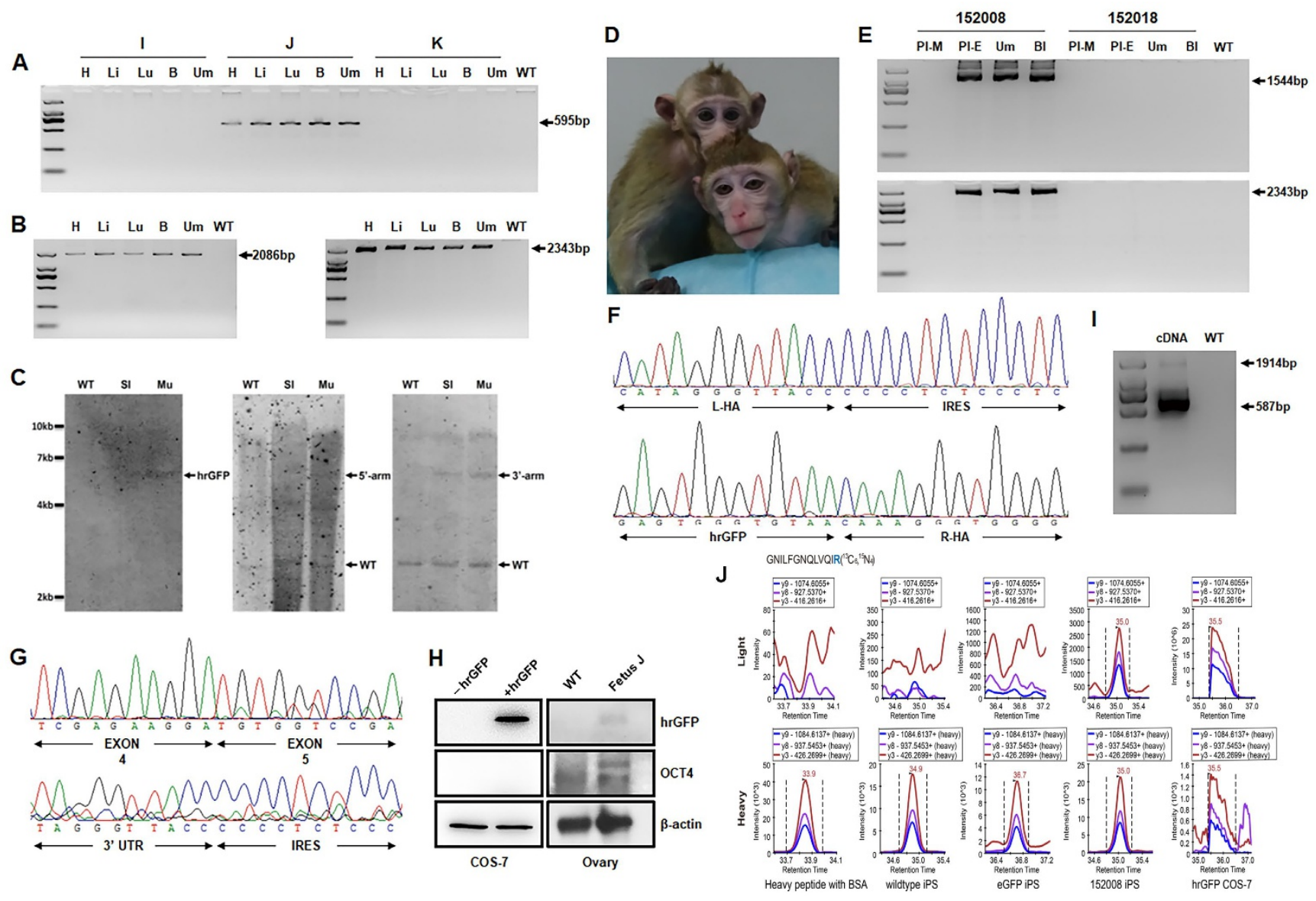

Figure 1 Generation of a precise Oct4-hrGFP knockin cynomolgus monkey model via CRISPR/Cas9-assisted homologous recombination. (A-C) Detection of Oct4-GFP knockin in abortive fetuses. (A) PCR amplification of a GFP fragment from different tissues of abortive fetuses I, J, K (060366A, B, and C). H, heart; Li, liver; Lu, lung; B, brain; Um, umbilical cord; WT, wild-type control. (B) PCR amplification of a 2086 bp 5'-arm with part of the GFP and the L-HA (left), or a 2343 bp 3'-arm with part of the GFP and the R-HA (right). (C) Southern blot analysis of the targeted allele. Stul-digested genomic DNA from small intestine and muscle of fetus J (060366B) was hybridized with different probes (left: Probe 1; middle: Probe 2; right: Probe 3). Expected fragment sizes: $4719 \mathrm{bp}$ (mutant), 3 $392 \mathrm{bp}$ (wild type). hrGFP, mutant fragment detected by hrGFP internal probe (Probe 1); 5'-arm, mutant fragment detected by 5 ' external probe (Probe 2); 3'-arm, mutant fragment detected by 3' external probe (Probe 3). WT, wild-type genomic DNA (negative control); Mu, muscle; SI, small intestine. (D-F) Detection of Oct4-GFP knockin in founder animals. (D) Photos of 403-day-old founder 152008 (left) and 401-day-old founder 152018 (right). (E) Nested PCR amplification of a 1544 bp 5'-arm with part of the GFP and the L-HA (upper), and a 2343 bp 3'-arm with part of the GFP and the R-HA (lower) from different tissues of founder animals (152008, 152018). $\mathrm{PI}-\mathrm{M}$, maternal portion of the placenta; PI-E, embryo portion of the placenta; Um, umbilical cord; BI, blood; WT, wild-type control. (F) The sequence of the amplified genomic DNA from the boundaries of 5'-arm (upper) and 3'-arm (lower). (G) The sequence of cDNA from the boundaries of Exons 4 and 5 (upper) as well as 3'-UTR and IRES (lower). (H) Western blot analysis of the expression of hrGFP and Oct4. The expression of hrGFP and Oct4 was examined in the ovaries of wild-type control and fetus J (060366B). COS7 cells with or without hrGFP overexpression serve as controls and $\beta$-actin was used as a loading control. (I) PCR products of cDNA reversely transcribed from mRNA isolated from the iPSCs of Founder 152008. WT, wild-type somatic cell control. (J) Extracted ion chromatograms of transitions monitored for detection of hrGFP protein in iPSCs generated from founder 152008 and different types of control by mass spectrometry. GNILFGNQLVQIR derived from hrGFP protein along with internal heavy standards were detected. Heavy peptide with BSA, isotope-labeled peptides were spiked into tryptic peptides of bovine serum albumin; wild-type iPS, iPS cells derived from wild-type monkey; GFP iPS, iPS cells with GFP overexpression; 152008 iPS, iPS cells derived from hrGFP knockin monkey 152008; hrGFP COS-7, COS-7 cells with hrGFP overexpression as a positive control. All primers used for PCR amplification were illustrated in Supplementary information, Figure S1 and described in Supplementary information, Table S4.

several modifications, including -2 and -5 bp deletions, were also detected in the placenta, umbilical cord and blood of Founder 152008 (Supplementary information,
Figure S4B and Table S5), indicating the mosaicism for CRISPR/Cas9-mediated precise editing.

The same mutation ( -2 bp deletion) and precise 
Oct4-hrGFP homologous recombination were detected by further genotyping analysis in the ovary of fetus $\mathrm{J}$ (Supplementary information, Figure S5 and Table S3), demonstrating the successful integration of Oct4-hrG$F P$ in ovaries of the fetuses via CRISPR/Cas9-mediated genome targeting. Excitingly, a weak Oct4-hrGFP band along with a wild-type Oct4 band were visualized by RT-PCR (Supplementary information, Figure S5D), indicating that the Oct4-hrGFP was precisely edited and expressed in germ cells. The result was further confirmed by sequencing the cDNA product, showing normal splicing between exons and precise IRES-hrGFP tagging (Figure $1 \mathrm{G}$ and Supplementary information, Data S8). Furthermore, hrGFP was specifically detected by western blot in the ovary of aborted fetus J, and expression of Oct4 was also detected as expected (Figure 1H). Taken together, $h r G F P$ has been precisely inserted into the Oct4 locus and expressed in the female germline.

Next, whole-genome sequencing of the knockin monkey (152008) and its parents (father, 071717; mother, 070952) was performed at a depth of about $55-65 \times$ to recover variants of lower frequencies (Supplementary information, Table S6). The genome was analyzed by SpeedSeq pipeline to screen small insertions and deletions (indels) and large indels using freebayes and lumpy methods [6], respectively (Supplementary information, Table S7). For sites with up to 5 mismatches, only one off-target 2 bp deletion was detected (Supplementary information, Figure S6, Tables S5 and S8). Whole-genome indel analysis with manual inspection revealed 6 founder-specific small indels and 4 large indels with split reads unique to the founder. One small indel was verified as on-target deletion (Supplementary information, Figure $\mathrm{S7}$ ), another $2 \mathrm{bp}$ indel as off-target deletion as mentioned above (Supplementary information, Figure S6), and a 920 bp large indel as on-target deletion by PCR and sequencing (Supplementary information, Table S8 and Data S9). Taken together, the precise knockin of $h r G F P$ cassette into Oct4 gene was verified by whole-genome sequencing. Six and thirteen split reads were detected to support 5' and $3^{\prime}$ end of the $h r G F P$ cassette, respectively, with one portion mapped on the chromosome 4 and the rest derived from $5^{\prime}$ or $3^{\prime}$ end of the $h r G F P$ cassette (Supplementary information, Figures S8 and S9). These observations were consistent with the above PCR analysis of precise $h r G F P$ knockin cassette.

The Oct4-hrGFP knockin model provides a favorable tool to study reprogramming, because Oct4 induction is a gold standard for full reprogramming [7]. Fibroblasts from ear biopsy of Founder 152008 were reprogrammed to induced pluripotent cells (iPSCs), which carried the same 2 bp deletion and exhibited the same homologous recombination of $h r G F P$ as Founder 152008 (Supplementary information, Figure S10A-S10C). Positive Oct4 immunostaining (Supplementary information, Figure S10D) showed successful induction and reprogramming in all iPSCs [7]. A weak Oct4-hrGFP cDNA band was detectable in sorted GFP-positive iPSCs by RT-PCR (Figure 1I), and sequencing showed normal splicing between exons and precise IRES-hrGFP tagging (Supplementary information, Figure S10E and Data S10). To further characterize the knockin allele, hrGFP protein in iPSCs was analyzed by selected reaction monitoring (SRM) mass spectrometry. With the heavy peptide internal standards labeled by stable isotopes, we observed two different light hrGFP tryptic peptides from iPSCs with the same elution time and approximately same relative SRM peak intensity ratios across multiple transitions as heavy internal standards (Figure 1J and Supplementary information, Figure S11), indicating the expression of hrGFP protein in the iPSCs. We conclude that the $h r G F P$ knockin does not interfere with Oct4 induction and hrGFP was co-expressed with Oct4 following reprogramming to pluripotency. Nevertheless, the weak hrGFP signal reminds us that better tagging is needed.

In summary, this study represents the first successful precise gene editing in primates, and also demonstrates minimal off-target effects of the CRISPR/Cas9 system in this species. Furthermore, this Oct4-hrGFP knockin model provided a versatile tool for primate reprogramming study.

Materials and Methods are available in Supplementary information, Data S1.

\section{Acknowledgments}

We thank members of Ji, Sha and Huang labs for helpful discussions. We are grateful to Dr Tian Chi (ShanghaiTech University) for excellent manuscript editing. This works is supported by the National Natural Science Foundation of China (81671516, U1302227, U1602224 and 31571534), the National Key R\&D Program (2016YFA0500903, 2016YFA0503300, 2016YFA0101401 and and 2017YFA0103803) and Local Grants (SKLRM-K201502 and 2015FA037).

Yiqiang Cui ${ }^{1, *}$, Yuyu Niu ${ }^{2,4,}$, Jiankui Zhou ${ }^{3, *}$, Yongchang Chen ${ }^{2,4}$, , Yiwei Cheng ${ }^{1}$, Shangang $\mathrm{Li}^{5}$, Zongyong $\mathrm{Ai}^{2}$, Chu $\mathrm{Chu}^{2}$, Hong $\mathrm{Wang}^{2}$, Bo Zheng ${ }^{1}$, Xuejin Chen ${ }^{5}$, Jiahao Sha ${ }^{1}$, Xuejiang Guo ${ }^{1}$,

Xingxu Huang ${ }^{3}$, Weizhi $\mathrm{Ji}^{2,4}$

${ }^{l}$ State Key Laboratory of Reproductive Medicine, Department of Histology and Embryology, Nanjing Medical University, 101 Longmian Rd., Jiangning District, Nanjing, Jiangsu 211166, China; ${ }^{2}$ Yunnan Key Laboratory of Primate Biomedicine Research; Institute of Primate Translational Medicine, Kunming University of Science and Technology, Kunming, Yunnan 
650500, China; ${ }^{3}$ School of Life Science and Technology, ShanghaiTech University, 100 Haike Rd., Pudong New District, Shanghai 201210, China; ${ }^{4}$ Kunming Ennovate Institute of Bioscience, Kunming, Yunnan 650000, China; ${ }^{5}$ Department of Laboratory Animal Science, School of Medicine, Shanghai Jiao Tong University, Shanghai 200025, China

*These four authors contributed equally to this work.

Correspondence: Weizhi Ji ${ }^{\mathrm{a}}$, Xingxu Huang ${ }^{\mathrm{b}}$, Xuejiang Guo

${ }^{a}$ E-mail: wji@1pbr.cn

bE-mail: huangxx@shanghaitech.edu.cn

cE-mail: guo_xuejiang@njmu.edu.cn

\section{References}

1 Phillips KA, Bales KL, Capitanio JP, et al. Am J Primatol 2014; 76:801-827.

2 Kim H, Kim JS. Nat Rev Genet 2014; 15:321-334.

3 Niu Y, Shen B, Cui Y, et al. Cell 2014; 156:836-843.

4 Ma Y, Zhang X, Shen B, et al. Cell Res 2014; 24:122-125.

5 Saitou M, Miyauchi H. Cell Stem Cell 2016; 18:721-735.

6 Chiang C, Layer RM, Faust GG, et al. Nat Methods 2015; 12:966-968.

7 Wernig M, Meissner A, Foreman R, et al. Nature 2007; 448:318-324.

(Supplementary information is linked to the online version of the paper on the Cell Research website.) 